\title{
Modal frequency and shape curvature as a measure of implant fixation: a computer study on the acetabular cup
}

\author{
Petr Henyš $\check{\mathrm{a}}^{\mathrm{a}}$, Steven Leuridan ${ }^{\mathrm{b}}$, Quentin Goossens ${ }^{\mathrm{b}}$, Michiel Mulier ${ }^{\mathrm{b}}$, Leonard Pastrav ${ }^{\mathrm{b}}$, Wim \\ Desmet $^{\mathrm{b}}$, Jos Vander Sloten ${ }^{\mathrm{b}}$, Kathleen Denis ${ }^{\mathrm{b}}$, Lukáš Čapek ${ }^{\mathrm{a}}$ \\ ${ }^{a}$ Technical University of Liberec, Department of Technologies and Structures, Studentská 1402/2, 46117 Liberec, Czech \\ Republic \\ ${ }^{b}$ KU Leuven, Department of Mechanical Engineering, Celestijnenlaan 300C, 3001 Leuven, Belgium
}

\begin{abstract}
Modal parameters are often investigated in order to assess the initial fixation of an implant. Most of studies are focused on the natural frequencies and frequency response function. Usually the femoral stem is tested although the acetabular cup fixation is important as well. The results of implant stability assessment are inconsistent and seem to suggest that frequency as a stability indicator is not sufficiently sensitive. In this study the sensitivity of the modal properties to changes in the bone-implant interface was investigated with the help of the finite element method (FEM). A novel fixation index based on modal shape curvature was investigated as a potential measure of the implant fixation. Modal frequencies are sensitive to interface changes in some manner, but suffer from insensitivity to local changes at bone-implant interface. The sensitivity up to $44 \%$ of natural frequencies to stiffness change due insertion steps was observed. The tested damage indicators are able to detect localized small changes in peripheral stiffness (5\% stiffness reduction) with $95 \%$ confidence under the noise up to $1 \%$. The modal shapes and their curvatures have a great potential to be a robust fixation indicator.
\end{abstract}

Keywords: Acetabular Cup, Initial Fixation, Finite Element Method, Modal Shapes, Damage Index

\section{Introduction}

It is generally known that insufficient initial fixation of press-fitted acetabular implants leads

3 to an excessive implant movement and consequent fibrous layer formation [43], which can cause

Email address: petr.henys@tul.cz (Petr Henyš)

Preprint submitted to Medical Engineering $\mathcal{E}$ Physic

June 18, 2018 
4 aseptic loosening of the implant [15, 14]. On the other hand, excessive mechanical press-fit fix-

5 ation leads to an inadequate stress load in the peripheral bone. This may result in damage to the

6 surrounding bone and subsequent implant failure [7]. Achieving the optimal fixation level is a

fundamental factor for the long term outcome of the implant longevity. Initial implant fixation

assessment is often based on destructive mechanical tests. Implant fixation can be measured

9 by pull-out [37], lever-out [27] or torsional [10] tests, which are all unfortunately restricted to

10 be used in an in vitro setting. Alternatively, medical imaging techniques can provide qualita-

11 tive information about the implant state, but cannot quantify the amount of the initial fixation.

12 Other major drawbacks of imaging techniques are; the emission of harmful rays, cumbersome

13 to be used per-operatively and diffraction phenomena due to presence of metal materials [16].

14 Alternative concepts to assess implant fixation based on vibrational methods have been widely

15 investigated by many authors (changes in natural frequencies or changes in the magnitude of

16 frequency response functions (FRF) [6, 12, 21]). An instrumented impact hammer for measuring

the implant stability has been developed and extensively tested. The impact hammer benefits

18 from time-domain feature from force cell mounted on the hammer while does not require the

19 response from bone itself [34, 4, 26, 33, 31]. Most of these studies are mainly concerned about

20 the hip stem, although acetabular component failure rates are similar or even slightly higher.

${ }_{21}$ Moreover the qualitative assessment of the initial stability by auditory or other cues is more

22 difficult than for the stem [24, 22, 38]. The monitoring of initial implant fixation by tracking

${ }_{23}$ frequency resonance shifts works well for dental implant [28, 29, 30], however the clinical im-

24 plementation in total hip replacement (THR) is still a challenge. Monitoring of initial fixation

25 has shown promising results on artificial and cadaveric bones and even on limited per operative

${ }_{26}$ trials on patients [12, 42, 32, 33, 18]. Challenges are still abundant however; patient-specific or

${ }_{27}$ operational factors may thwart the interpretation of changes in the FRFs. Additionally there is

28 still no consistent opinion whether the lower or higher frequency band is more sensitive to initial

29 fixation.

so To investigate isolated parameters that influence the vibrational behaviour of the bone-implant

${ }_{31}$ system, FEM models have been developed. Pastrav et al investigated the influence of contact

32 conditions on the resonance frequencies of a hip stem by FEM. They found that change at con-

33 tact area correlates with frequency shifts and more complex modes are more sensitive to changes

${ }_{34}$ in contact area [40, 21]. The higher sensitivity of more complex modes has been supported by 
Qi et al [44].

Modal analysis is the most widely used method to assess implant fixation, but only the natural frequencies are commonly studied despite the fact that they are rather global and would not reflect adequately local changes in the bone-implant interface. Corresponding mode shapes are more interesting because of their spatial dependence which potentially is more influenced by local changes in the bone-implant interface. Moreover, the curvature of mode shape is often used in industrial engineering to localize and quantify structural damage [8, 56, 39]. A curvature-based damage index (DI) can be calculated based on the difference or ratio of two states, a healthy and damaged state. The DI approximates the strain energy changes due to local changes in structural stiffness. Since the initial fixation of an implant is directly related to the interface stiffness, characterizing the implant stability using a form of this DI could be of interest. Based on the state-of-the art knowledge of the current methods dealing with monitoring of implant fixation, this study aims to investigate computationally the sensitivity of the frequencies, mode shapes and their curvatures to bone-acetabular implant interface changes. To the best of our knowledge, such study has never been published and brings a fresh look to the usability of a vibration approach in monitoring the fixation of implants

\section{Materials and Methods}

\subsection{FE Models}

The numerical model was composed of a validated finite element of an artificial pelvic bone. The finite element model of the bone was validated with respect to its modal properties. The material properties of the model were optimized based on the experimental modal analysis and large scale gradient based optimization scheme with accuracy error below 1\% [17]. The implant model was based on a type SF-58 acetabular implant (Beznoska, Czech Republic). An isotropic, homogeneous material model was assumed for the implant with a Young's modulus of 110 [GPa] (TI6Al4V), a Poisson's ratio of $0.3[-]$ and a density of $4500 \mathrm{~kg} \cdot \mathrm{m}^{-3}$. The bone-implant interface was modelled with simple interface conditions without friction or contact definitions. The bone and implant were kinematically constrained in order to prevent relative movement between them. The change at the interface was generated by different shared contact areas (SCA) at different implantation depths. The depth of reamed cavity was varied from 4.2 to $-2.3 \mathrm{~mm}$ and was defined as a polar gap (Figure 1). The range was carefully set-up so that at the lowest value the implant 
does not go through the medial wall while at the same time the implant only made contact with

66 completely uncovered trabecular bone. The highest range $4.2 \mathrm{~mm}$ was selected so that the cortical

67 contact area was more than $1000 \mathrm{~mm}^{2}$. No Dirichlet boundary conditions were assumed to ensure

68 no influence of boundary conditions. The hemispherical cup was positioned at $45^{\circ}$ abduction and $25^{\circ}$ anteversion [51]. The following simplifications are taken into account:

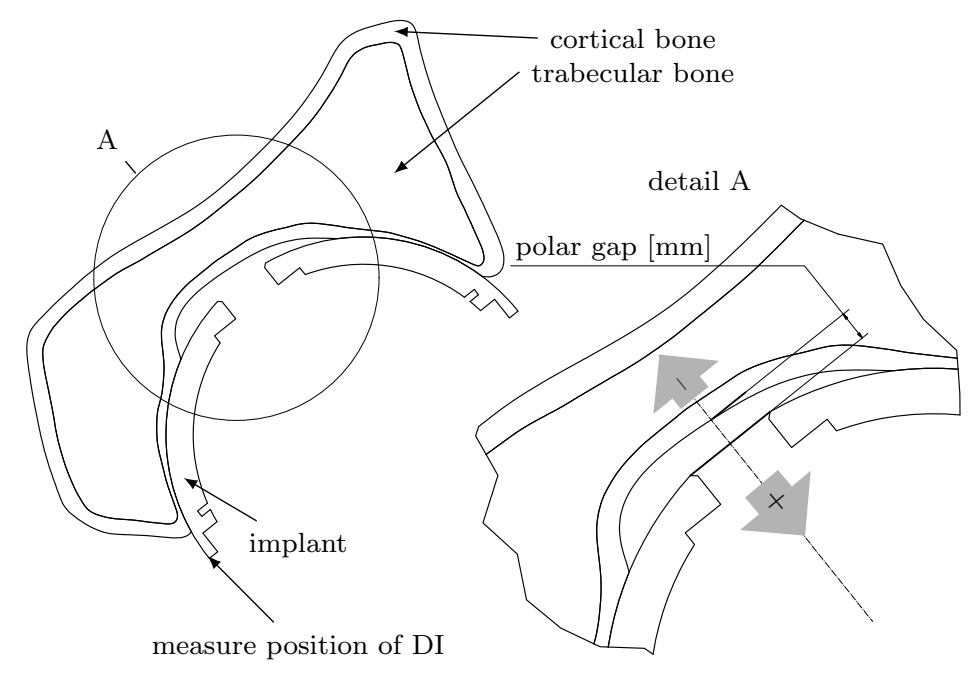

Figure 1: An illustrative cut of pelvic bone-acetabular implant interface with polar gap imposed. A positive/negative polar gap refers to the depth of reamed cavity that is lower/higher than the depth of acetabular notch.

- Simplified interface model was described by changing of contact area without relative displacement between the bone and implant.

- No friction or stiffening effects were considered in the model.

- No damping properties were taken into account.

74 2.2. Modal Analysis

A Krylov-Schur based method for solving the generalized eigenvalue problem was used from 76 the library SLEPc [19]. Thirty-five eigenvalues and corresponding mode shapes were computed

77 in a frequency range of $0-8 \mathrm{kHz}$ by a spectral shift-invert approach. The factorization solver used

78 in the transformation was of a MUMPS type [2]. The full numerical scheme was implemented 
in Python/C with parallel execution of assembling and solving the eigenvalue problem using

${ }_{80}$ the PETSc/SLEPc library [3]. The convergence of eigenvalues was verified on a parametrized

${ }_{81}$ mesh according to [17]. A mesh convergence analysis was carried out on the implant mesh.

82 The maximum difference between eigenvalues was set to 5\%. This threshold was satisfied by an

83 average element size of $1 \mathrm{~mm}$. Displacement field was approximated by a piecewise quadratic

${ }_{84}$ shape function. The mesh was created using the CGAL-3D library [53].

${ }_{85}$ In order to compare two mode shapes quantitatively, the Modal Assurance Criterion (MAC) was

${ }_{86}$ used. The MAC is a standard similarity criterion commonly used in experimental and numerical

${ }_{87}$ modal analysis to match two mode shapes [1, 48]. The MAC is defined as:

$$
\operatorname{MAC}\left(\psi_{1}, \psi_{2}\right)=\frac{\left|\psi_{1}^{T} \psi_{2}\right|^{2}}{\psi_{1}^{T} \psi_{1} \psi_{2}^{T} \psi_{2}}
$$

s8 Where ${ }^{T}$ stands for the transpose and $\psi_{1}, \psi_{2}$ the real mass normalized mode shape vectors.

89 To take into account the minor changes of the mesh for every insertion step, the mode shape

90 vectors were interpolated on a template mesh with radial basis interpolation method [57]. The

91 interpolation error was established by testing different mesh densities. The interpolation error

92 was set to $10^{-6}$ and the MAC error to $1 \%$. These criteria were satisfied with average element size

93 $1 \mathrm{~mm}$ obtained from convergence analysis.

94 2.3. Damage Indices

With all mode shapes computed, a curvature based method commonly used in structural health monitoring for damage identification was applied in order to quantify the changes of mode

shapes for points at the bone-implant peripheral interface. The curvature was measured on the peripheral rim of acetabular implant at finite element nodes (Figures 1 and 2). The curvature $k$ of the $i^{\text {th }}$ mode shape $\psi_{i}$ defined as second order derivation of $\psi$ with respect to spatial coordinates:

$$
\kappa_{i}=\frac{\partial^{2} \psi_{i}}{\partial \mathbf{x}^{2}}, \mathbf{x}=\{x, y, z\}
$$

was computed with second order forward differentiation. Three Damage Indices (DI-A, DI-B and DI-C) were used. The damage indices were sensitive to stiffness changes commonly occurring as a result from damage to a structure. Due to the reduced stiffness of a damaged object, the local curvature of the mode shape increases. The damage indices compare quantities derived from the mode shapes of the bone-implant construct between different states of the bone-implant 
construct. The undamaged state of the bone-implant construct was defined as configuration with the implant at an insertion level with depth $-2.3 \mathrm{~mm}$. This state represents the ideal fixation level of the implant. The damaged states of the bone-implant constructs were defined as the rest of the insertion levels. The damaged and undamaged states are indicated by superscripts $\cdot u$, un respectively. The curvatures $\kappa$ are expressed as a function of peripheral angle $\phi$ defined in Figure 2. The variable $N$ introduces the number of modal shapes used to compute the DI and has value 10 unless otherwise specified.

The first damage indicator (DI-A) [5] was defined as the difference between the mode shape curvatures $\kappa$ of a damaged and undamaged state of the bone-implant construct:

$$
\delta_{A}(\phi)=\frac{1}{N} \sum_{i=1}^{N}\left\|\left[1+\frac{\Delta \boldsymbol{\kappa}_{\phi, i}}{\max \left(\Delta \boldsymbol{\kappa}_{i}\right)-\min \left(\Delta \boldsymbol{\kappa}_{i}\right)}\right]^{2}\right\|
$$

with curvature difference $\Delta \boldsymbol{\kappa}_{\phi, i}=\boldsymbol{\kappa}_{\phi, i}^{d}-\boldsymbol{\kappa}_{\phi, i}^{u}$. The DI-A was normalized with respect to the max/min values of the mode shape curvature difference. The second damage index DI-B [13] was based on the fractional relation of the modal energy $\mathrm{U}$ between the damaged and undamaged state of the bone-implant construct.

$$
\delta_{B}(\phi)=\frac{\sum_{i=1}^{N} \frac{U_{i, \phi}^{d}}{U_{i}^{d}}}{\sum_{i=1}^{N} \frac{U_{i, \phi}^{u}}{U_{i}^{u}}}
$$

where $U_{i, \phi}$ is proportional to the modal strain energy of $i^{\text {th }}$ mode shape [52]:

$$
U_{i, \phi} \propto I N T E R^{\phi \leftarrow b c^{e}} \int_{\Omega_{e}}\left[\kappa_{x}^{2}+\kappa_{y}^{2}+\kappa_{z}^{2}\right] d \Omega_{e}
$$

where $\kappa_{x}, \kappa_{y}, \kappa_{z}$ express the partial curvatures with respect to coordinates. The modal energy of $i^{\text {th }}$ mode shape is computed over elements on the peripheral rim of the implant. In order to compare it with nodal valued indexes DI-A, DI-C, elemental modal strain energy $U_{e, i}$ was interpolated to a nodal space at barycentric coordinates $\left(b c^{e}\right)$ of $e^{\text {th }}$ element. The total modal strain energy $U_{i}$ of $i^{\text {th }}$ modal shape is a sum over the elements. The third damage index DI-C was based on the norm of the difference of the mode shape curvature $\kappa$ corresponding to a damaged and undamaged state:

$$
\delta_{C}(\phi)=\frac{1}{N} \sum_{i=1}^{N}\left\|\left(\boldsymbol{\kappa}_{i, \phi}^{d}\right)^{2}-\left(\boldsymbol{\kappa}_{i, \phi}^{u}\right)^{2}\right\|
$$

where the $\kappa_{i, \phi}$ is the mode shape curvature of $i^{\text {th }}$ shape at peripheral angle $\phi$. For every DI the average over the modal shapes $N$ was calculated. Subsequently, the DI for every mode was 
normalized as follows:

$$
\hat{\delta}(\phi)=\frac{\delta(\phi)-\mu_{\delta}}{\sigma_{\delta}}
$$

where $\mu$ and $\sigma$ are the mean over angle $\phi$ and standard deviation respectively. For values $\hat{\delta}(\phi)>2$ the presence of the damage at measured angle $\phi$ was presumed. This threshold value corresponds to a probability at the $97.725 \%$ confidence level (assuming a normal distribution) that damage was located at the identified location [9, 54, 13, 45, 46, 50].

\subsection{Computational Experiment with the Damage Indices}

In order to investigate the potential of the defined DIs to distinguish between damaged and undamaged states, firstly an additional computational experiment was performed before testing the DIs on the pelvic bone model. A simplified FEM model was created consisting only of the acetabular cup itself. In order to simulate the localized damage, the so called 'damage segment' is introduced in Figure 2. The damage segment contains the reduced Young's modulus to simulate the damage.

The parametrized hemispherical cup was created using the CGAL library and was meshed using linear tetrahedral elements with an average element size of $1 \mathrm{~mm}$ (the thickness of cup was $4 \mathrm{~mm}$ ). The displacement of the cup was approximated by quadratic basis functions.

\subsubsection{Sensitivity of DI to Change of Stiffness}

The stiffness of the cup was then changed locally at several angles $\phi$ by modifying the modulus of elasticity at these angles. Angle $\phi$ and Youngs modulus $E$ were parametrized in a following way to assess the DI performance:

- Parameter $E$ : steps 5,25 and 50\% at the indicated locations,

- Parameter $\phi$ : varying from 0 to $300^{\circ}$ with step $60^{\circ}$ (6 location in total)

The segment width $d$ was $5^{\circ}$. A total of 18 simulations were performed.

\subsubsection{Sensitivity of DI to a Noise}

In order to assess the DIs performance with noisy data, the modal shapes were contaminated by noise controlled by variable $\sigma$ according to:

$$
\hat{\psi}=\psi\left[1+\alpha\left(0, \frac{\sigma}{100}\right)\right]
$$




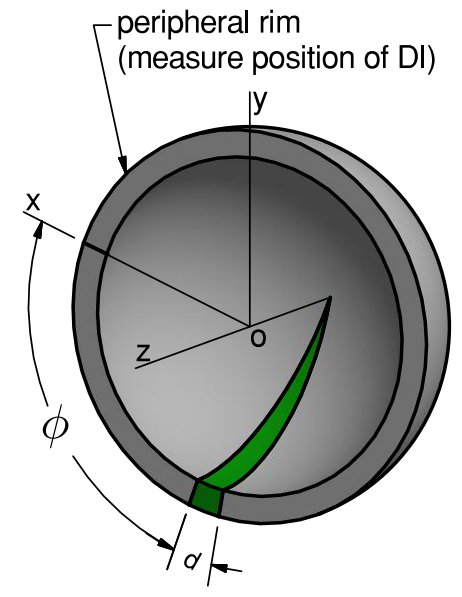

Figure 2: Parametrized acetabular cup with damage segment (green color), which has the position measured by angle $\phi$ $\left[{ }^{\circ}\right]$ with respect to global coordinate system $O$ and arc-length $d\left[^{\circ}\right]$. 


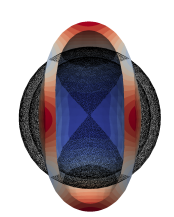

\# 1

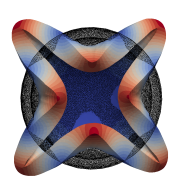

\# 6

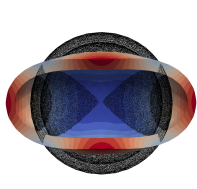

\# 2

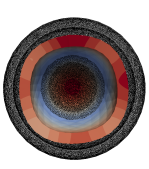

\# 7

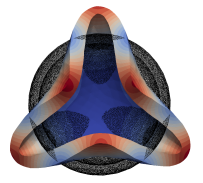

\# 3

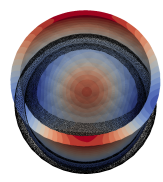

\# 8

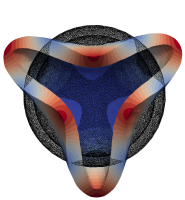

\# 4

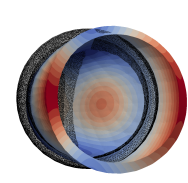

\# 9

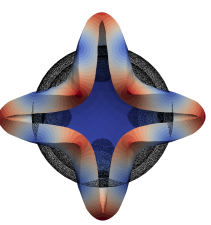

\# 5

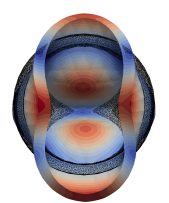

\# 10

Figure 3: Modal shapes of acetabular cup

corresponding to these mode shapes are shown in Table 1 The first natural frequencies and corresponding modal shape are similar to the results reported by [20]. The frequency range of the first natural frequencies of the cup was considerably higher than the bone itself or of the bone-implant assembly. Figure 4 shows the values obtained for the different DIs in function of the parametrized angle and Youngs modulus. With a reduction of 5\% of the Youngs modulus, only the DI-A identified the correct angles where the Youngs modulus was reduced. When the reduction of the Youngs modulus was higher, the amplitudes of DI generally increased except for DI-B, where the results do not show a general trend. Table 2 shows the noise sensitivity of every DI with damage at an angle of $0^{\circ}$ and with all ten mode shapes included. The results for other damage locations were similar. The results listed in the table clearly indicated that DI-A performed well and could detect the smallest change in Youngs modulus with noise around 1\% 
Table 1: Natural frequencies of acetabular cup without Young's modulus reduction

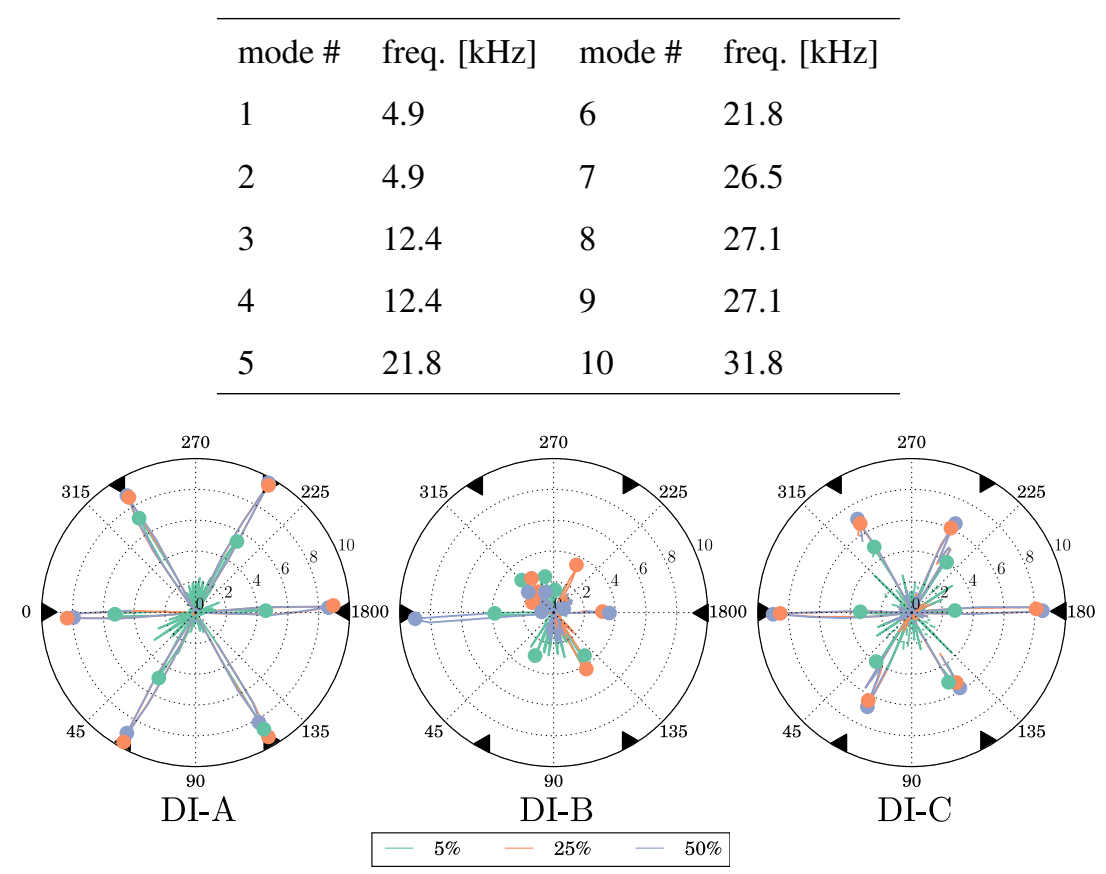

Figure 4: A typical evaluation of DI magnitude for three reduction levels of Young's modulus (5, 25 and $50 \%)$ and for six locations of damage segment(black triangles: $\left.\phi=\{0,60,120,180,240,300\}\left[^{\circ}\right]\right)$. The models $\mathrm{A}, \mathrm{B}, \mathrm{C}$ refer to a different DI formulation.

while the highest change in Youngs modulus could be detected with 5\% noise at the 95\% con-

fidence level. The DI-B can detect only changes with noise at the $1 \%$ level and fails to detect damage with noise at the $5 \%$ level.

\subsection{Pelvic Bone-implant Model}

The changes at the interface are described by the evaluation of the SCA at different cavity depths. The evolution of the SCA is presented in Figure 5. The evolution of the contact area is split into two bone regions. When inserted in the cavity with the lowest polar gap, the implant is mainly in contact with the trabecular bone. From the first stages of insertion, corresponding to a higher polar gap, to the final stages of insertion, the cortical area increases until it reaches the maximal value $(1.98 \mathrm{~mm})$, after the maximum of cortical area is reached, the cortical area decreases while the trabecular contact increases. The difference of the natural frequencies between 
Table 2: Sensitivity of DIs to a noise (a success detection of damage $>95 \%$ )

\begin{tabular}{llll}
\hline & \multicolumn{3}{c}{ maximum noise [\%] } \\
Damage [\%] & DI-A & DI-B & DI-C \\
\hline 5 & 1 & - & 0.5 \\
25 & 3 & 0.5 & 1 \\
50 & 5 & 0.5 & 3 \\
\hline
\end{tabular}

the first and final insertion step is shown in Table 3. The Table contains the maximal differ-

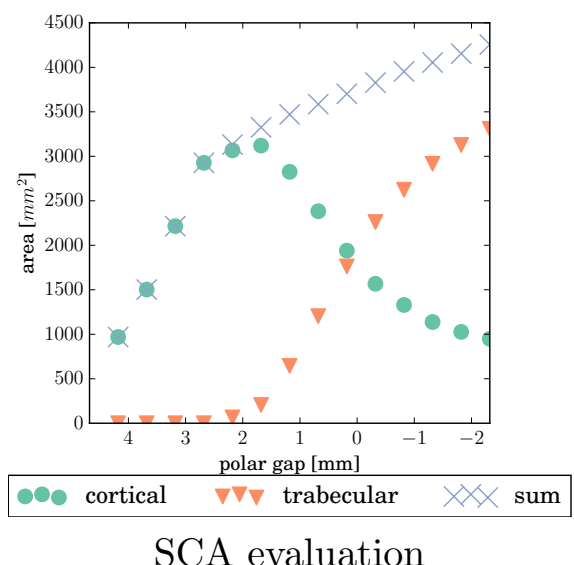

SCA evaluation

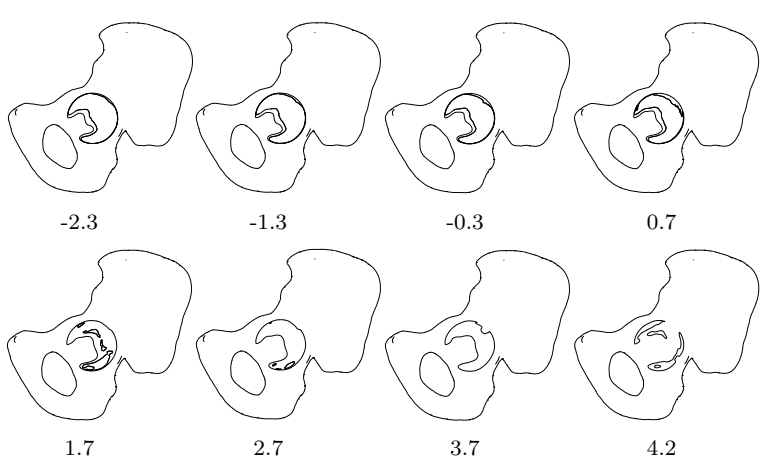

Illustrative view of SCA evaluation

Figure 5: Evaluation of SCA during different insertion levels

190

ences only for the most sensitive natural frequencies.All differences exceeded 35\%. According to Figure 6 the relative sensitivity of frequencies decreased with higher modes. All first twenty flexible modes however have sensitivities higher than $20 \%$. The shapes associated with the five most sensitive frequencies are shown in Figure 7 from high to low sensitivity. The first mode has mainly a torsional character; however the rest of the modes significantly deforms around the acetabular rim and behaves as a combination of bending and torsional modes. The sensitivity of the shapes is shown in Figure 8 . The first and second chart present the global change of minimum or maximum of MAC values due to changes of the SCA. When the modal complexity increased, the difference in minimal MAC values between successive insertion steps increased and the maximal MAC values decreased. The last chart shows the MAC evolution between each 


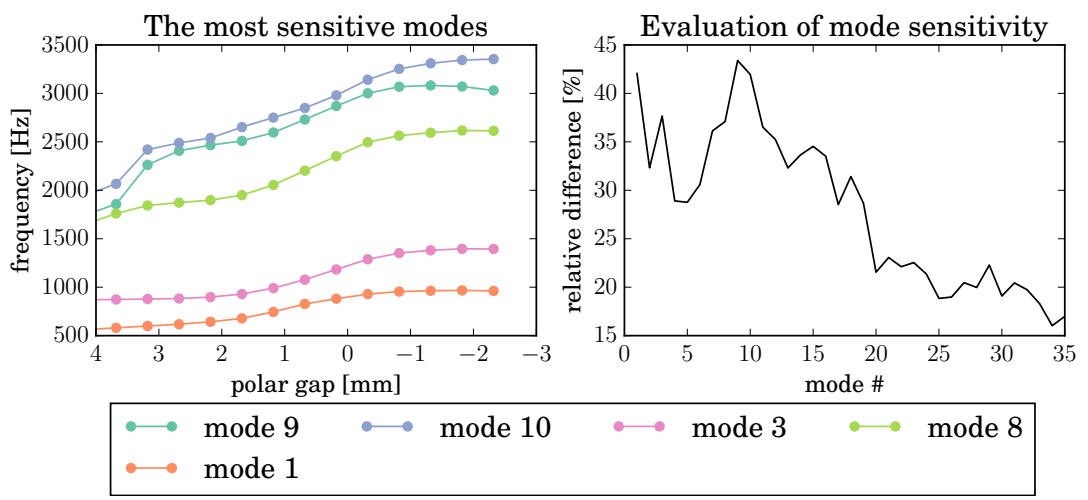

Figure 6: Frequency sensitivity

Table 3: Selected sensitivity of natural frequencies

\begin{tabular}{llllll}
\hline mode \# & 9 & 10 & 3 & 8 & 1 \\
\hline rel. difference [\%] & 43.3 & 42.1 & 41.9 & 37.7 & 37.1 \\
\hline
\end{tabular}

201 insertion step for the mode shapes associated with the most sensitive frequencies. Although the 202 most sensitive frequencies varied more than $35 \%$, only shapes 8,9 and 10 varied significantly ${ }_{203}$ (with a minimal MAC of $0.731,0.712$ and 0.662 respectively). 


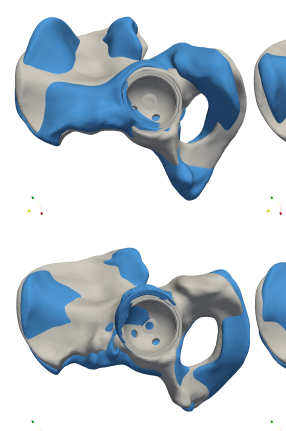

\# 9
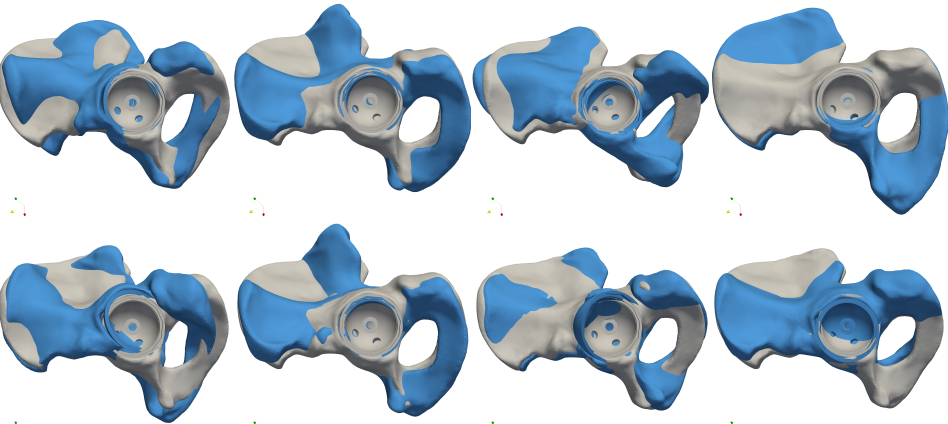

\# 10

\# 3

\# 8

\# 1

Figure 7: Associated shapes with the most sensitive frequencies, top line: $-2.3 \mathrm{~mm}$, bottom-line: $4.2 \mathrm{~mm}$. Grey and blue colours represent the undeformed and deformed geometry respectively.
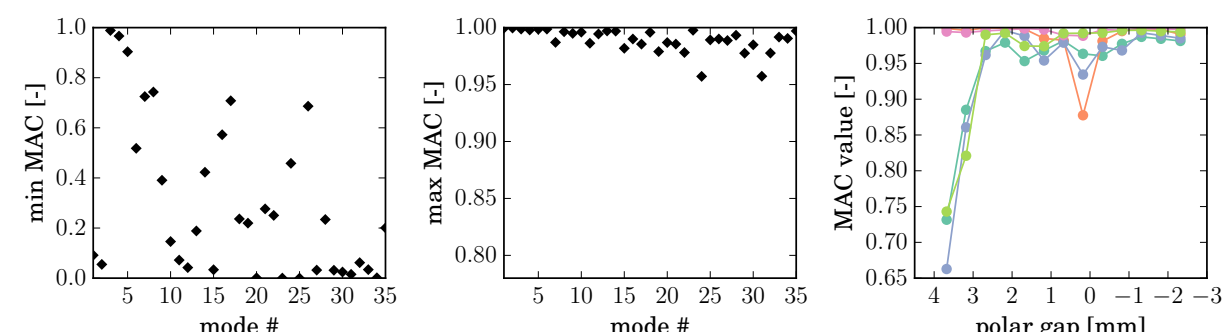

$\because \quad$ shape 9

Figure 8: MAC evaluation over the range of modes 

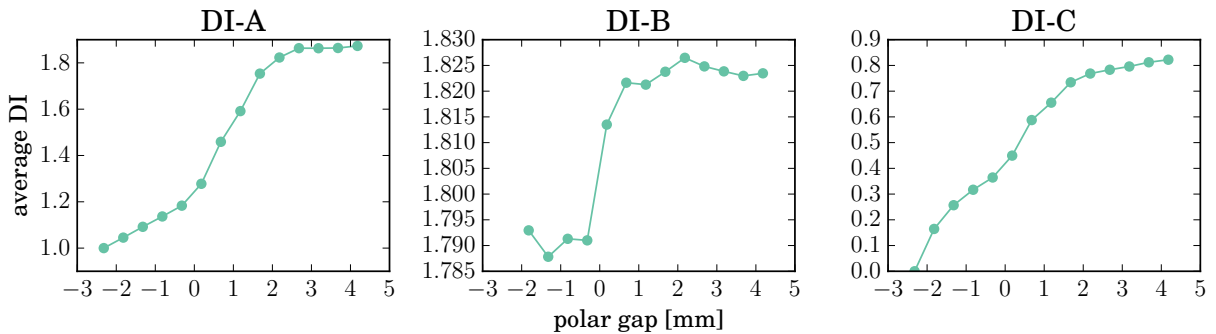

Figure 9: Average value of non-normalized DI over whole peripheral rim. 


\section{Discussion}

The presented study investigated the dynamic behaviour of the bone-implant system for different contact conditions between bone and acetabular implant. These contact conditions correlate well to the initial stability of the implant. The study of interface evaluation revealed that during the insertion phase, different amounts of cortical and trabecular bone came into contact with the implant. The evolution of the SCA indicated that the ratio of cortical and trabecular bone in contact with the implant evolved non-linearly. In addition possible large variation due to other factors (cup position and orientation, acetabular shape, bone quality-mechanical properties) may additionally influence this distribution. Evaluating the dependency of modal parameters on the SCA, significant sensitivity of the resonance frequencies was found. The natural frequencies changes ranged from 0 to $44 \%$. This seems to point to the frequencies to be a promising predictor of the implant stability; however some important remarks must be made. To the best of the authors knowledge, there is no experimental study that indicated such high sensitivity. Typically, the frequency shift of some modes is around $10-20 \%$, albeit with no guarantee that the modes were properly tracked. Tracking resonance frequencies by correlating mode shapes between successive steps is important to understand possible mode switching and veering of the system [25]. Tracking correlated mode shapes as the structure changes has not been performed in biomechanical studies, although it can completely distort the obtained frequency shifts. In this work, the modal frequencies were carefully tracked by comparing the MAC criteria of each mode in order to exclude possible mismatches. Mode switching and veering can occur if the changes in structure are too large. Moreover, the modal density is quite high in the selected frequency range and the modes could easily switch or veer. Roughly speaking, to use shifts in natural frequencies in order to assess the implant fixation, experimental modal analysis with more than one measuring point is likely advised for systems with a high modal density. The first two natural frequencies of the acetabular cup/bone system are in range $4-5 \mathrm{kHz}$, which is in accordance with numerical study at the time domain [35]. Although the natural frequencies of the pelvic bone itself are lower ([17, 36]), due to significantly higher stiffness of the cup implant and its smaller dimension than pelvic bone, the bone-implant assembly (with fully evolved contact interface) becomes stiffer and thus natural frequencies shift to higher frequency band.

The mode shapes in this study were assessed as potential predictor of implant fixation. However, the shapes need to be measured carefully, especially as the more complex modes are hard 
to acquire and would indeed benefit from sophisticated measuring equipment such as a laser vibrometry scanner. The sensitivity of thirty-five modal shapes was investigated in this study. It was shown that the accuracy of more complex mode shapes decreased. From a practical point of view, only the first ten modes can likely be taken as a predictor. The higher modes are more sensitive to noise and small, rather insignificant, changes in the model as illustrated by the MAC evaluation of the mode shapes (Figure 8). The higher spread of MAC values with higher modes was due to the computational shape accuracy and sensitivity to model parameters. From a practical point of view, measurement of the mode shapes is important in order to properly pair and compare shifts in natural frequencies between successive insertion steps. Once these mode shapes are available, implant fixation can be evaluated in terms of changes in the peripheral stiffness which can be deducted from changes in these mode shapes. In this study some alternative indicators to determine initial fixation were introduced. Changes in interface stiffness were linked to changes in mode shape curvatures. It is generally known that higher curvature values reflect lower local stiffness. Three different indicators based on the curvature were numerically tested using an artificial acetabular cup to assess the indicators basic performance. Results showed that the most promising indicator is the ratio between two elastic energies (expressed as the square of the mode shape curvature) comparing the actual and undamaged reference states (DI-A, the reference state: $-2.3 \mathrm{~mm}$ polar gap). A second indicator (DI-B) demonstrated the least interesting performance and was deemed not suitable to be used as a stability predictor. The last measure DI-C was able to detect changes in stiffness with a lower accuracy. The accuracy of the different DIs was tested on mode shapes contaminated by different levels of uniform noise. The minimal detection level was achieved only for the DI-A and DI-C with stiffness changes of at least $5 \%$ with maximal noise of $1 \%$ and $0.5 \%$ respectively. The performance of the DI is sensitive to the accurate computing of the second order derivation (or the first order derivative of the strain) of the mode shapes. Since the computing of second order derivative of shapes are susceptible to data noise, computing by wavelet or another approximation method would be an important step to clinically implement the proposed DIs [11, 49].

Numerical experiments on simplified cup showed a nearly perfect detection ability of the DIs, although frequency changes were limited to only 5\%. In that case the interpretation of DI is clear. Interpretation of the DI results for the bone-implant system was more difficult. Although it could detect changes in the peripheral acetabular stiffness, it was not possible to determine the 
state of initial fixation based on only one peak in the polar DI graph but rather needed to evaluate the shape. Additionally the behaviour of the DIs was not monotonic which indicated that the stiffness was changing in different ways during the insertion steps. Future work will focus on determining how the resulting stiffness evolves during insertion using a virtual torque testing and will correlate these results with the DIs obtained. In this work the insertion level with polar gap $-2.3 \mathrm{~mm}$ was taken as the undamaged reference state (the most stable implant configuration). In a clinical situation, the evolution of implant fixation should be measured between steps and the initial and final step could be compared. This study has several limitations. A first limitation is the description of the bone-implant interface. The bone and implant are kinematically constrained. These constraints are more representative for the fixation the implant would obtain after the osseointegration process. As a numerical modal analysis expects linear behaviour of the model, implementation of non-linear contact conditions would inhibit computing the modal parameters in an efficient way. Nevertheless, the influence of non-linear interface conditions could be explored for a limited set of cases by included them in the FE model by local approximation techniques (harmonic balance method [55, 47]). The resultant frequency response would likely be strongly non-linear close to a nearly zero initial fixation of the implant. This method will be implemented into the model in future studies. It must be pointed out that the non-linear interface behaviour is usually omitted because the behaviour of the bone-implant model during the insertion is more or less linear when measured between insertion steps except when the implant would be in clinically unstable state due to insufficient fixation during insertion or bone fractures that may occur during insertion [48, 6, 21, 23, 41]. A second limitation or challenge of the proposed fixation monitoring approach is to develop a clinical device that would be able to measure the mode shapes of acetabular cup experimentally during surgery. From a technical point of view, the experimental device should be able to measure the mode shapes by applying a controlled excitation to the acetabular cup and measure its response at a certain number of locations, preferably on the peripheral rim of the cup. Possible measurement solutions could include the use of some compact laser vibrometry or a high speed camera which provide a field of harmonic displacements. Repeated measurements for every insertion step will likely be necessary to average out the noise in the mode shape extraction process (identification and computation of the shape curvature). Another limitation of the computational model can be seen in the material model used. The bone visco-elasticity affects the implant in two folds: it adds a viscous damping into 


\section{Competing Interests}

325

\section{Conclusion} properties.

\section{Funding}

None

\section{Ethical Approval}

Not required

modal dynamics and thus complicates the evaluation of the natural frequencies and shapes; due

to stress relaxation of bone, the initial implant stability might vary in the time.

From a clinical point of view, the measuring of the initial fixation during the implantation step can help to objectively achieve an optimal press-fit fixation without exceeding the biomechanical limits of the bone and implant. A vibration based approach, such as the one presented in this study, furthermore has the advantage that the low level of vibrations needed to perform the measurement will not damage or change the interface while measuring.

In this study the authors presented a new method to measure implant fixation by modal shape based damage indicator and assessed its potential by computer experiments on a simplified acetabular cup model and on a validated finite element model. The next step would be the experimental application of the proposed method on composite and cadaver bones. Simultaneously, advanced computational model of bone-implant construct with complex interface properties will be developed in order to gain deeper knowledge how to describe the implant fixation by modal

The authors have no conflict of interest in relation to the present study. 
Appendix A. Full-field Results on Pelvis
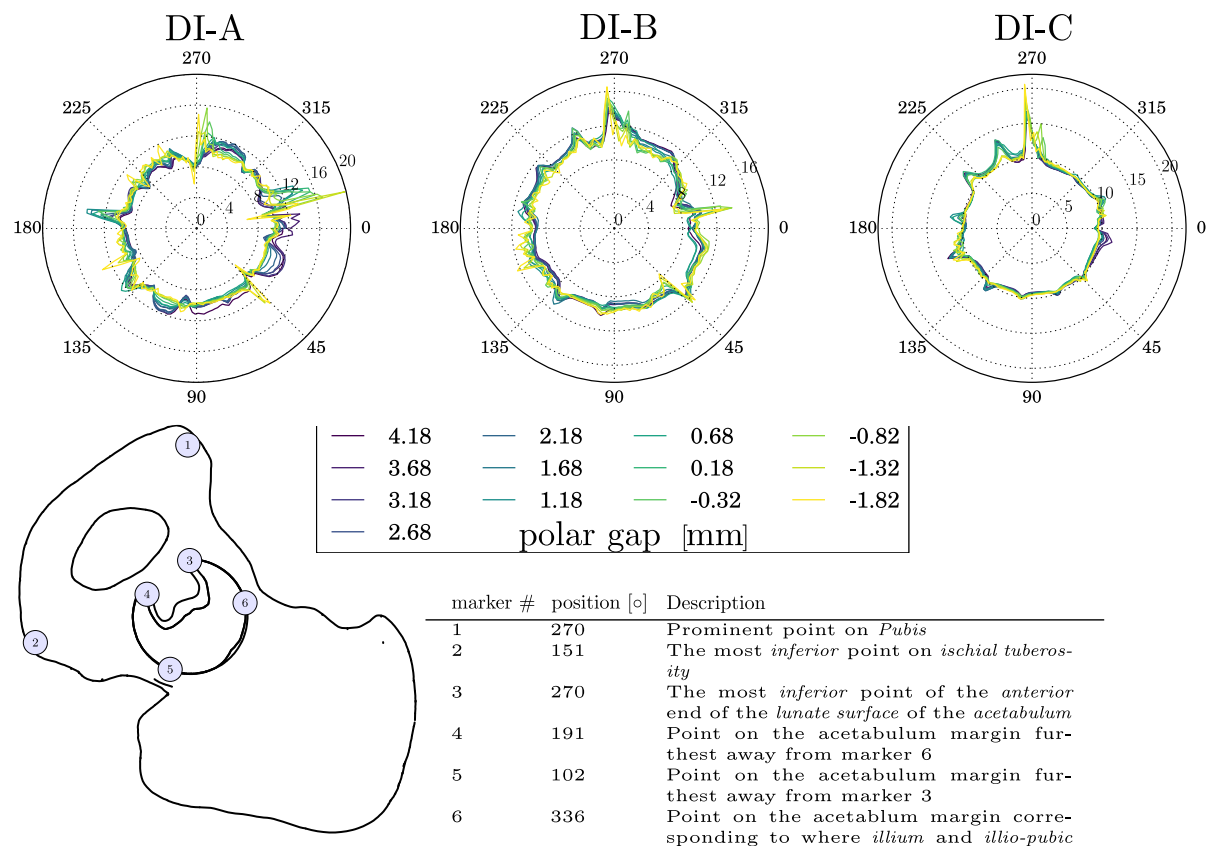

$2.18-0.68$
$-\quad 1.68$

0.18

-0.82
-1.32

- $1.18-0.32$ polar gap $[\mathrm{mm}]$

$-1.82$

marker \# position [o] Description

\begin{tabular}{lll}
\hline 1 & 270 & Prominent point on Pubis \\
2 & 151 & The most inferior point on ischial tuberos-
\end{tabular}

The most inferior point of the anterior end of the lunate surface of the acetabulum Point on the acetabulum margin furPoint on the acetabulum

thest away from marker 3 margin fur-

Point on the acetablum

sponding to where illium and illio-pubic ramus meet

Figure A.10: A typical evaluation of damage index during insertion steps. Coloured curves express the value of DI at actual insertion step and at position on implant rim. The curves contains a radial offset of value 10 in order to get figure readable. The polar graph axes correspond to acetabular rim and its centre. 


\section{References}

[1] Allemang, R. J., 2003. The modal assurance criterion-twenty years of use and abuse. Sound and vibration 37 (8), $14-23$.

[2] Amestoy, P. R., Duff, I. S., Koster, J., L’Excellent, J.-Y., 2001. A fully asynchronous multifrontal solver using distributed dynamic scheduling. SIAM Journal on Matrix Analysis and Applications 23 (1), 15-41.

[3] Balay, S., Abhyankar, S., Adams, M. F., Brown, J., Brune, P., Buschelman, K., Dalcin, L., Eijkhout, V., Gropp, W. D., Kaushik, D., Knepley, M. G., McInnes, L. C., Rupp, K., Smith, B. F., Zampini, S., Zhang, H., 2015. PETSc Web page.

[4] Bosc, R., Tijou, A., Rosi, G., Nguyen, V.-H., Meningaud, J.-P., Hernigou, P., Flouzat-Lachaniette, C.-H., Haiat, G., 2018. Influence of soft tissue in the assessment of the primary fixation of acetabular cup implants using impact analyses. Clinical Biomechanics 55, 7-13.

[5] Chandrashekhar, M., Ganguli, R., 2009. Damage assessment of structures with uncertainty by using mode-shape curvatures and fuzzy logic. Journal of Sound and Vibration 326 (3), 939-957.

[6] Cristofolini, L., Varini, E., Pelgreffi, I., Cappello, A., Toni, A., 2006. Device to measure intra-operatively the primary stability of cementless hip stems. Medical engineering \& physics 28 (5), 475-482.

[7] Curtis, M., Jinnah, R., Wilson, V., Hungerford, D., 1992. The initial stability of uncemented acetabular components. Journal of Bone \& Joint Surgery, British Volume 74 (3), 372-376.

[8] Dawari, V., Vismawala, G., 2013. Structural damage identification using modal curvature differences. Journal of Mechanical and Civil Engineering., 2278-1684.

[9] Fan, W., Qiao, P., 2009. A 2-d continuous wavelet transform of mode shape data for damage detection of plate structures. International Journal of Solids and Structures 46 (25), 4379-4395.

[10] Fehring, K. A., Owen, J. R., Kurdin, A. A., Wayne, J. S., Jiranek, W. A., 2014. Initial stability of press-fit acetabular components under rotational forces. The Journal of arthroplasty 29 (5), 1038-1042.

[11] Fu, C.-L., Feng, X.-L., Qian, Z., 2010. Wavelets and high order numerical differentiation. Applied Mathematical Modelling 34 (10), 3008-3021.

[12] Georgiou, A., Cunningham, J., 2001. Accurate diagnosis of hip prosthesis loosening using a vibrational technique. Clinical Biomechanics 16 (4), 315-323.

[13] Grooteman, F., 2012. Damage detection and probability of detection for a shm system based on optical fibres applied to a stiffened composite panel. ISMA2012, Leuven, 17-19.

[14] Hamilton, W. G., Calendine, C. L., Beykirch, S. E., Hopper, R. H., Engh, C. A., 2007. Acetabular fixation options first-generation modular cup curtain calls and caveats. The Journal of arthroplasty 22 (4), 75-81.

[15] Healy, W. L., Casey, D. J., Iorio, R., Appleby, D., 2002. Evaluation of the porous-coated anatomic hip at 12 years. The Journal of arthroplasty 17 (7), 856-863.

[16] Hecht, S., Adams, W. H., Narak, J., Thomas, W. B., 2011. Magnetic resonance imaging susceptibility artifacts due to metallic foreign bodies. Veterinary Radiology \& Ultrasound 52 (4), 409-414.

[17] Henyš, P., Čapek, L., Feb 2017. Material model of pelvic bone based on modal analysis: a study on the composite bone. Biomechanics and Modeling in Mechanobiology 16 (1), 363-373.

[18] Henyš, P., Čapek, L., Apr 2018. Impact force, polar gap and modal parameters predict acetabular cup fixation: A study on a composite bone. Annals of Biomedical Engineering 46 (4), 590-604. 
[19] Hernandez, V., Roman, J., Tomas, A., Vidal, V., 2007. Krylov-schur methods in slepc. Universitat Politecnica de Valencia, Tech. Rep. STR-7.

[20] Hothan, A., Huber, G., Weiss, C., Hoffmann, N., Morlock, M., 2011. Deformation characteristics and eigenfrequencies of press-fit acetabular cups. Clinical Biomechanics 26 (1), 46-51.

[21] Jaecques, S., Pastrav, C., Zahariuc, A., Van der Perre, G., 2004. Analysis of the fixation quality of cementless hip prostheses using a vibrational technique. In: Proceedings of ISMA 2004 International Conference on Noise and vibration engineering: 20-22 September 2004; Leuven. pp. 443-456.

[22] Köster, G., Munz, D., Köhler, H.-P., 1993. Clinical value of combined contrast and radionuclide arthrography in suspected loosening of hip prostheses. Archives of orthopaedic and trauma surgery 112 (5), 247-254.

[23] Lannocca, M., Varini, E., Cappello, A., Cristofolini, L., Bialoblocka, E., 2007. Intra-operative evaluation of cementless hip implant stability: A prototype device based on vibration analysis. Medical engineering \& physics 29 (8), 886-894.

[24] Lieberman, J., Huo, M., Schneider, R., Salvati, E., Rodi, S., 1993. Evaluation of painful hip arthroplasties. are technetium bone scans necessary? The Journal of bone and joint surgery. British volume 75 (3), 475-478.

[25] Mace, B. R., Manconi, E., 2011. Dispersion phenomena in coupled waveguides: veering, locking and strong coupling effects.

[26] Mathieu, V., Michel, A., Lachaniette, C.-H. F., Poignard, A., Hernigou, P., Allain, J., Haiat, G., 2013. Variation of the impact duration during the in vitro insertion of acetabular cup implants. Medical Engineering \& Physics 35 (11), 1558-1563.

[27] Meneghini, R. M., Meyer, C., Buckley, C. A., Hanssen, A. D., Lewallen, D. G., 2010. Mechanical stability of novel highly porous metal acetabular components in revision total hip arthroplasty. The Journal of arthroplasty 25 (3), $337-341$.

[28] Meredith, N., Alleyne, D., Cawley, P., 1996. Quantitative determination of the stability of the implant-tissue interface using resonance frequency analysis. Clinical oral implants research 7 (3), 261-267.

[29] Meredith, N., Books, K., Fribergs, B., Jemt, T., Sennerby, L., 1997. Resonance frequency measurements of implant stability in viva. a cross-sectional and longitudinal study of resonance frequency measurements on implants in the edentulous and partially dentate maxilla. clinical oral implants research 8 (3), 226-233.

[30] Meredith, N., Shagaldi, F., Alleyne, D., Sennerby, L., Cawley, P., 1997. The application of resonance frequency measurements to study the stability of titanium implants during healing in the rabbit tibia. Clinical oral implants research 8 (3), 234-243.

[31] Michel, A., Bosc, R., Mathieu, V., Hernigou, P., Haiat, G., 2014. Monitoring the press-fit insertion of an acetabular cup by impact measurements: Influence of bone abrasion. Proceedings of the Institution of Mechanical Engineers, Part H: Journal of Engineering in Medicine 228 (10), 1027-1034.

[32] Michel, A., Bosc, R., Meningaud, J.-P., Hernigou, P., Haiat, G., 11 2016. Assessing the acetabular cup implant primary stability by impact analyses: A cadaveric study. PLOS ONE 11 (11), 1-14.

[33] Michel, A., Bosc, R., Sailhan, F., Vayron, R., Haiat, G., 2016. Ex vivo estimation of cementless acetabular cup stability using an impact hammer. Medical Engineering \& Physics 38 (2), 80-86.

[34] Michel, A., Bosc, R., Vayron, R., Haiat, G., 2015. In vitro evaluation of the acetabular cup primary stability by impact analysis. Journal of Biomechanical Engineering 137 (3), 031011. 
[35] Michel, A., Nguyen, V.-H., Bosc, R., Vayron, R., Hernigou, P., Naili, S., Haiat, G., May 2017. Finite element model of the impaction of a press-fitted acetabular cup. Medical \& Biological Engineering \& Computing 55 (5), $781-791$.

[36] Neugebauer, R., Werner, M., Voigt, C., Steinke, H., Scholz, R., Scherer, S., Quickert, M., 2011. Experimental modal analysis on fresh-frozen human hemipelvic bones employing a $3 \mathrm{~d}$ laser vibrometer for the purpose of modal parameter identification. Journal of Biomechanics 44 (8), 1610-1613.

[37] Olory, B., Havet, E., Gabrion, A., Vernois, J., Mertl, P., 2004. Comparative in vitro assessment of the primary stability of cementless press-fit acetabular cups. Acta Orthopædica Belgica 70 (1), 31-37.

[38] Ovesen, O., Riegels-Nielsen, P., Lindequist, S., Jensen, I., Munkner, T., Torfing, T., Marving, J., 2003. The diagnostic value of digital subtraction arthrography and radionuclide bone scan in revision hip arthroplasty. The Journal of arthroplasty 18 (6), 735-740.

[39] Pandey, A., Biswas, M., Samman, M., 1991. Damage detection from changes in curvature mode shapes. Journal of sound and vibration 145 (2), 321-332.

[40] Pastrav, L., Devos, J., Van der Perre, G., Jaecques, S., 2009. A finite element analysis of the vibrational behaviour of the intra-operatively manufactured prosthesis-femur system. Medical engineering \& physics 31 (4), 489-494.

[41] Pastrav, L., Jaecques, S., Mulier, M., Der Perre, G. V., 2008. Detection of the insertion end point of cementless hip prostheses using the comparison between successive frequency response functions. Journal of Applied Biomaterials \& Biomechanics 6 (1).

[42] Pastrav, L. C., Jaecques, S. V., Jonkers, I., Van der Perre, G., Mulier, M., 2009. In vivo evaluation of a vibration analysis technique for the per-operative monitoring of the fixation of hip prostheses. Journal of orthopaedic surgery and research 4 (1), 10.

[43] Pilliar, R., Lee, J., Maniatopoulos, C., 1986. Observations on the effect of movement on bone ingrowth into poroussurfaced implants. Clinical orthopaedics and related research 208, 108-113.

[44] Qi, G., Mouchon, W. P., Tan, T. E., 2003. How much can a vibrational diagnostic tool reveal in total hip arthroplasty loosening? Clinical Biomechanics 18 (5), 444-458.

[45] Rucevskis, S., Janeliukstis, R., Akishin, P., Chate, A., 2016. Mode shape-based damage detection in plate structure without baseline data. Structural Control and Health Monitoring.

[46] Rucevskis, S., Sumbatyan, M. A., Akishin, P., Chate, A., 2015. Tikhonov's regularization approach in mode shape curvature analysis applied to damage detection. Mechanics Research Communications 65, 9-16.

[47] Sanliturk, K., Ewins, D., 1996. Modelling two-dimensional friction contact and its application using harmonic balance method. Journal of sound and vibration 193 (2), 511-523.

[48] Scholz, R., Hoffmann, F., von Sachsen, S., Drossel, W.-G., Klöhn, C., Voigt, C., 2013. Validation of densityelasticity relationships for finite element modeling of human pelvic bone by modal analysis. Journal of biomechanics 46 (15), 2667-2673.

[49] Shao, X., Ma, C., 2003. A general approach to derivative calculation using wavelet transform. Chemometrics and Intelligent Laboratory Systems 69 (1-2), 157-165.

[50] Siebel, T., Friedman, A., Koch, M., Mayer, D., 2012. Assessment of mode shape-based damage detection methods under real operational conditions. In: 6th European Workshop on Structural Health Monitoring. Dresden, Germany. pp. $1-8$. 
[51] Spears, I. R., Pfleiderer, M., Schneider, E., Hille, E., Bergmann, G., Morlock, M. M., 2000. Interfacial conditions between a press-fit acetabular cup and bone during daily activities: implications for achieving bone in-growth. Journal of biomechanics 33 (11), 1471-1477.

[52] Stubbs, N., Kim, J., Topole, K., 1992. An efficient and robust algorithm for damage localization in offshore platforms. In: Proceedings of the ASCE tenth structures congress. pp. 543-546.

[53] The CGAL Project, 2015. CGAL User and Reference Manual, 4.7 Edition. CGAL Editorial Board. URL http://doc.cgal.org/4.7/Manual/packages.html

[54] Tomaszewska, A., 2010. Influence of statistical errors on damage detection based on structural flexibility and mode shape curvature. Computers \& structures 88 (3), 154-164.

[55] Von Groll, G., Ewins, D. J., 2001. The harmonic balance method with arc-length continuation in rotor/stator contact problems. Journal of sound and vibration 241 (2), 223-233.

[56] Wahab, M. A., De Roeck, G., 1999. Damage detection in bridges using modal curvatures: application to a real damage scenario. Journal of Sound and Vibration 226 (2), 217-235.

[57] Walt, S. v. d., Colbert, S. C., Varoquaux, G., 2011. The numpy array: A structure for efficient numerical computation. Computing in Science \& Engineering 13 (2), 22-30. 